\title{
Date and Time of Death
}

National Cancer Institute

\section{Source}

National Cancer Institute. Date and Time of Death. NCI Thesaurus. Code C117450.

The date and time of death. 